essential operators in implementing strategic action plans to practice.

Conclusions Strong collaboration and communication is needed in order to decrease the home and leisure injuries. The aim is to increase the discussion about the possibilities in preventing the injuries.

\section{9 USING RAI-HC ASSESSMENT INSTRUMENT TO CLASSIFY HOME CARE CLIENTS IN FINLAND BASED ON FIRE EMERGENCY EVACUATION CAPACITY}

${ }^{1}$ Magnus Biörkgren, ${ }^{1}$ Frank Borg, ${ }^{2}$ Seppo Männikkö, ${ }^{3}$ Leila Mäkinen, ${ }^{4}$ Vesa-Pekka Tervo. ${ }^{1}$ University of Jyväskylä, Finland; ${ }^{2}$ Tampere Region Emergency Services, Finland; ${ }^{3}$ City of Tampere, Finland; ${ }^{4}$ Keski-Uusimaa Department for Rescue Services, Finland

\subsection{6/injuryprev-2016-042156.409}

Background When an uncontrollable fire is detected in a building there may be a 2-3 minutes window for the occupants to move to safety. Poor physical function and cognition may become critical factors in an evacuation situation. In this study a novel functional scale measuring emergency evacuation capacity of home care clients is presented.

Methods A fire security expert visited about 250 home care clients assessing their evacuation capacities. For the evacuation capacity three categories were used: 1 ) is able to evacuate; 2) may be able; 3) unable to evacuate. This data was linked to comprehensive assessments of clients functional and health status performed by home care nurses using the interRAI Home Care Assessment Instrument (RAI-HC). The goal was to investigate whether the evacuation capacity classification can be explained in terms of RAI-HC variables. As the mathematical method we have employed "classification and regression trees" (CART).

Results A fire evacuation capacity scale "EVAC" was developed using four levels of cognitive function as major categories. These categories were split into final groups based on performance in physical function. For each group we calculated the average capacity score ranging from 0 to 1 based on the dependent variable.

Conclusions The evacuation scale gives an estimate of a client's ability to get out in case of a fire. The scale can be used to single out high risk persons for which compensatory safety technology may be required. The scale could reduce costs in assessing evacuation capacity of home care clients.

\section{MOTORCYCLE TYPE, FAMILARITY AND RIDER AGE: A CONDITINAL PROCESS ANALYSIS}

${ }^{1}$ Brown Julie, ${ }^{2}$ Baldock Matthew, 'Albanese Bianca, 'Meredith Lauren, ${ }^{3}$ Fitzharris Michael, ${ }^{4}$ Robin Turner. ${ }^{1}$ NeuRA Injury Prevention Research Centre and UNSW NSW; ${ }^{2}$ CASR University of Adelaide; ${ }^{3}$ MUARC, Monash University; ${ }^{4}$ School of Public Health and Community Medicine, UNSW

\subsection{6/injuryprev-2016-042156.410}

Background Motorcyclists are vulnerable road users and with increasing registrations, the number of motorcyclists killed and injured continues to rise. Commonly reported risk factors for crash involvement include the type of motorcycle, the rider's familiarity with the motorcycle and rider age. However, identifying potential risk factors is only the first step. To develop effective interventions, there is a need to understand how risk factors work together. This paper aims to examine the relationship between the type of motorcycle, the rider's familiarity with the motorcycle and rider age as risk factors for crash involvement using a case control sample and conditional process analysis.

Methods A case control sample consisting of 100 seriously injured motorcyclists and 500 controls was collected in NSW, Australia between 2012 and 2014 using in-depth crash investigation and survey. Condiitonal process analysis was used to test a moderated mediation effect of key risk factors; motorcycle type (sports motercycle versus other), rider familiarity with the motorcycle ( $\mathrm{km}$ ridden on the motorcycle) and rider age (years) on crash involvement while controlling for gender and most common type of riding (recreation versus other). This was acheived using the PROCESS macro in SAS that implements a series of regression analyses to estimate direct and indirect effects of the risk factors and interactions, as well as testing the significance of these effects.

Results Riders of sports motorcycles were more likely to be in the crash sample than those riding other types of motorcycles, however this effect is mediated by the rider's familiarity with motorcycle. Furthermore, this indirect effect is moderated by rider age, with the effect being more pronounced in older riders. Conclusions This analysis provides the first insight into how commonly reported risk factors related to motorcycle type, familiarity with a motorcycle and rider age work together. Specifically, this analysis identifies high priority targets for interventions aimed at mitigating crash risk through these risk factors.

\section{Fire Safety and Burn Injuries}

\section{Post Mon 1.3}

\section{BURDEN OF FIRE-RELATED INJURIES IN FINLAND}

${ }^{1}$ Kari Haikonen, ${ }^{2}$ Pirjo Lillsunde, ${ }^{1}$ Anne Lounamaa, ${ }^{3}$ Philippe Lunetta, ${ }^{4}$ Jyrki Vuola. ${ }^{1}$ National Institute for Health and Welfare, Finland; ${ }^{2}$ Ministry of Social Affairs and Health; ${ }^{3}$ University of Turku, Finland; ${ }^{4}$ Helsinki Burn Centre, Finland

\subsection{6/injuryprev-2016-042156.411}

Background The aim of this research was to examine the burden of (severe) fire-related injuries in Finland.

Methods All together twelve separate data sets were gathered for conducting the study. Finnish Hospital Discharge register (FHDR) was the core data in which the injured persons could be identified. The Causes of Death register was the data to identify fire-related deaths. Criteria of inclusion for further study were that a person had been to inpatient care or died. Data on sickness allowances, different kinds of rehabilitation funding, (disability) pensions were obtained from the Social Insurance Institution of Finland. Other types of disability allowances or pensions were obtained from the Finnish Centre for Pensions. Causes of Death data supplemented with socioeconomic data were obtained from Statistics Finland. A data from Statutory Accident Insurance was obtained to cover work-related accidents. A five-year sample of patients with fire-related burn was obtained from the Helsinki Burn Centre. The Finnish Hospital Discharge Register was available at the National Institute for Health and Welfare, Finland. The whole study consisted of five sub-studies published in scientific journals.

Results Quality, usability and some methodological issues of using the FHDR were resolved. A descriptive epidemiological study on the injuries nationwide was conducted. Inpatient care costs were approximated nationwide. Indirect burden of fire-related deaths 